\title{
A TEMÁTICA FÍSICA-AMBIENTAL ABORDADA NOS ENCONTROS DE PRÁTICAS DE ENSINO DE GEOGRAFIA DA REGIÃO SUL
}

\author{
The physical and environmental theme approached in Meetings \\ of Southern Geography Teaching Practices \\ El tema físico-ambiental abordado en los Encuentros de Practicas \\ Enseñanza de Geografía de la Región Sul
}

\begin{abstract}
Éliton Paulo Novais ${ }^{*}$ José Edézio da Cunha**

*Mestrando no Programa de Pós-graduação em Geografia da Universidade Estadual do Oeste do Paraná Campus de Marechal Cândido Rondon - eliton.nvais@gmail.com.

**Professor/Orientador na Graduação e Pós-graduação em Geografia da Universidade Estadual do Oeste do Paraná - Campus de Marechal Cândido Rondono - edeziocunha@hotmail.com.
\end{abstract}

Recebido em 19/10/2019. Aceito para publicação em 20/10/2019.

Versão online publicada em 10/11/2019 (http://seer.ufrgs.br/paraonde)

\section{Resumo:}

Este artigo parte de uma pesquisa de mestrado em desenvolvimento no Programa de PósGraduação em Geografia da Unioeste de Marechal Cândido Rondon, e pretende analisar e entender, a partir das dissertações e teses produzidas, o que vem sendo pesquisado acerca do ensino de Geografia pelos Programas de Pós-Graduação Stricto Sensu em Geografia no estado do Paraná. O Ensino de Geografia é uma área em contínua construção e expansão dentro da Ciência Geográfica, neste sentido, o objetivo deste trabalho foi de analisar as publicações das quatro edições do Encontro de Práticas de Ensino de Geografia da Região Sul (2013, 2014, 2017 e 2018), em relação à temática física-ambiental no âmbito do Ensino de Geografia, se enquadrando assim nas pesquisas denominadas "Estado da Arte" (EAr). Para fazermos a coleta dos artigos estabelecemos que a busca dar-se-ia a partir da leitura dos resumos e palavras-chave. Porém, nas quatro edições do ENPEG-SUL diferentes critérios para a formatação dos artigos foram utilizados, assim nem todos os artigos apresentavam resumos e palavras-chave, desta forma, na ausência destes foram analisadas as introduções dos artigos e, quando necessário, também o trabalho completo. Entre a primeira e a quarta edição do Encontro de Práticas de Ensino de Geografia da Região Sul foram publicados 245 trabalhos, dos quais 82 ligados à temática física-ambiental. Onde as temáticas mais apresentadas/publicadas foram acerca dos Mapas/Cartografia, Educação Ambiental e TIC/Geotecnologias.

Palavras-chave: Ciência Geográfica. Eventos Científicos. Produção Científica. Ensino de Geografia.

\footnotetext{
Abstract:

This article is part of a master's degree research under development at Marechal Cândido Rondon's Geography Postgraduate Program, and it aims to analyze and understand, from the dissertations and theses produced, what has been researched about the teaching of geography by Stricto Sensu Graduate Programs in Geography in the state of Paraná. Geography Teaching is an area in continuous construction and expansion within Geographic Science. In this sense, the objective of this work was to analyze the publications of the four editions of the Southern Geography Teaching Practices Meeting (2013, 2014, 2017 and 2018), in relation to the physical-environmental theme in the context of Geography Teaching, thus fitting in the research field called "State of Art" (EAr). To collect the articles, we
} 
established that the survey would take place by reading the abstracts and keywords. However, in the four editions of ENPEG-SUL different criteria were used to format the articles, therefore, not all articles presented abstracts and keywords, so in theirs's absence, the introductions of the articles were analyzed and, when necessary, also the complete work. Between the first and the fourth edition of the Meeting of Geography Teaching Practices of the Southern Region, 245 works were published, of which 82 related to the physicalenvironmental theme. Where the most presented / published themes were about Maps / Cartography, Environmental Education and ICT / Geotechnologies.

Key-words:Geographic Science.Scientific Events. Scientific Production. Geography Teaching.

\begin{abstract}
Resumen:
Este artículo es parte de una pesquisa de maestría en desenvolvimiento del programa de la Escuela de Posgrado en Geografía de Unioeste de Marechal Cândido Rondon, en busca para analizar y comprender, basado en disertaciones y teses producidas, lo que viene siendo pesquisado acerca de la enseñanza de Geografía por los Programas de Posgrado Stricto Sensu en Geografía en el estado de Paraná. La enseñanza de Geografía es un campo en constante construcción y expansión dentro de la Ciencia Geográfica, a este respecto, el objetivo de este trabajo fue analizar las publicaciones de las cuatro ediciones del Encuentro de Practicas de Enseñanza de geografía de la Región Sul (2013, 2014, 2017 y 2018), relacionados a la temática físico-ambiental en el marco de la Enseñanza de Geografía, se encuadrando así las pesquisas designadas "Estado da Arte" (EAr). Para hacer la coleta de los artículos, empezaría con la búsqueda de la lectura de los informes y de las palabras-llaves. Sin embargo, en las cuatro ediciones de ENPEG-SUL, diferentes criterios fueran utilizados el en formato de los artículos, así, ni todos los artículos tenían informes o palabras-llaves, entonces, en sus ausencias, fueran analizadas las introducciones y cuándo necesario, el texto completo. Mientras la primera y la cuarta edición del Encuentro de las Pláticas de Enseñanza de Geografía de la Región Sul, fueran publicados 245 trabajos, a los cuales, 82 están relacionados con la física-ambiental. Donde las temáticas más presentadas/publicadas son acerca de Mapas/Cartografía, Educación Ambiental y TIC/Geotecnología.
\end{abstract}

Palabras-clave:Ciencia Geográfica. Eventos Ciêntíficos. Procucción Científica.Enseñanza de Geografía.

\title{
1 Introdução
}

A afirmativa de que vêm crescendo, nos últimos anos, o número de pesquisas voltadas ao Ensino de Geografia se deve ao "incremento de investigações na temática, envolvendo maior número de pesquisadores que através de suas dissertações e teses iniciam-se de modo mais formal na pesquisa sobre o ensino da nossa disciplina escolar" (CALLAl et al., 2016, p. 44).

O Encontro de Práticas de Ensino de Geografia da Região Sul (ENPEG-SUL) tem sido um importante evento de divulgação e discussão desta temática. Isso porque 0 mesmo tem o objetivo de trazer reflexões acerca do ensino de Geografia frente às mudanças do século XXI e assim, compreender o papel da Geografia no contexto da contemporaneidade. Trazendo à tona debates sobre as concepções, práticas e experiências de ensino, nos diferentes campos da Ciência Geográfica e buscando consolidar uma rede de troca de conhecimentos entre universidades, pesquisadores, professores, profissionais e alunos.

Cabe ressaltar, que o artigo, aqui apresentado, parte de uma pesquisa de

ParaOnde!?, Porto Alegre, v.12 n.2, p.107-114, 2019.http://seer.ufrgs.br/paraonde

Edição Especial - III Colóquio de Pesquisadores em Geografia Física Ensino de Geografia 
mestrado, em desenvolvimento no Programa de Pós-Graduação em Geografia da Universidade Estadual do Oeste do Paraná (Unioeste) - Campus de Marechal Cândido Rondon, que pretende analisar e entender o que vem sendo pesquisado acerca do ensino de Geografia nos Programas de Pós-Graduação Stricto Sensu em Geografia do estado do Paraná.

Diante do exposto e com o desenvolvimento desta pesquisa de mestrado percebemos a importância de analisarmos também o que vem sendo discutido nos eventos a respeito desta temática, tanto nacional como regionalmente, principal fato que justifica a apresentação deste artigo.

Por entendermos que o Ensino é uma área em contínua construção e expansão dentro da Geografia, definimos analisar e evidenciar o que foi publicado nas quatro edições (2013, 2014, 2017 e 2018) do ENPEG-SUL em relação à temática físicaambiental no âmbito do Ensino de Geografia.

A escolha da utilização do termo "Temática Física-Ambiental" ao invés do termo "Temática Físico-Naturais", o qual já se encontra bastante difundido por pesquisadores da área, se deu pelo fato de que nesta terceira edição do "Colóquio de Pesquisadores em Geografia Física e Ensino de Geografia", o termo foi tratado desta maneira.

Este estudo, desenvolvido a partir do levantamento das publicações do ENPEGSUL, se enquadra nas pesquisas denominadas "Estado da Arte" (EAr). As quais, segundo Fonseca, Salvi e Torres (2014, p. 143), "de forma geral, possuem uma ideia em comum: mapear a produção acadêmica nas mais diversas áreas do conhecimento. Onde após esse mapeamento abre-se, naturalmente, debate acerca do tema levantado".

Cabe ressaltar, que as pesquisas do tipo "estado da arte" podem ser enquadradas tanto no método qualitativo como no quantitativo. Já que, por meio de seu mapeamento permitem destacar elementos, seja em conjunto ou individualmente, acerca do que vem sendo produzido cientificamente (FREITAS, 2015).

Buscando definir o tempo e o espaço do trabalho, recorremos aos anais das quatro edições do ENPEG-SUL: 2013, 2014, 2017 e 2018, com a finalidade de mapear os trabalhos voltados à temática física-ambiental no contexto do Ensino de Geografia.

Para fazermos a coleta dos artigos relacionados à temática definida, estabelecemos que a busca dar-se-ia a partir da leitura dos resumos e palavras-chave destes artigos. Porém, nas quatro edições do ENPEG-SUL diferentes critérios para a formatação dos artigos foram utilizados, assim nem todos os artigos apresentavam resumos e palavras-chave, desta forma, na ausência destes foram analisadas as introduções dos artigos e, quando necessário, também o trabalho completo.

\section{Desenvolvimento}

O primeiro ENPEG-SUL, realizado em 2013 em Porto Alegre/Rio Grande do Sul, na Universidade Federal do Rio Grande do Sul (UFRGS), teve como tema o "Ensino de Geografia no Contemporâneo: Metodologias e Vivências Escolares", e registrou 64 trabalhos publicados, cabe ressaltar que nesta edição os trabalhos não foram divididos por grupos.

ParaOnde!?, Porto Alegre, v.12 n.2, p.107-114, 2019.http://seer.ufrgs.br/paraonde Edição Especial - III Colóquio de Pesquisadores em Geografia Física Ensino de Geografia 
Dos 64 trabalhos apresentados, 27 abordaram sobre a temática física-ambiental, a saber: Educação Ambiental, TIC/Geotecnologias, Mapas/Cartografia, Clima, Paisagem Natural, Geologia e conceitos gerais de Geografia Física, conforme podem ser conferidos no Quadro 1 a seguir:

Quadro 1 - ENPEG-SUL (2013).

\begin{tabular}{|c|c|}
\hline Tema abordado & № de trabalhos \\
\hline Educação Ambiental & 9 \\
\hline TIC/Geotecnologias & 8 \\
\hline Mapas/Cartografia & 4 \\
\hline Clima & 2 \\
\hline Paisagem Natural & 2 \\
\hline Geologia & 1 \\
\hline $\begin{array}{c}\text { Geografia Física (Conceitos } \\
\text { Gerais) }\end{array}$ & 1 \\
\hline
\end{tabular}

Fonte: Anais do Encontro de Práticas de Ensino da Região Sul, 2013. Organização: Os autores.

A segunda edição do ENPEG-SUL, realizada em 2014 em Florianópolis/Santa Catarina, na Universidade Federal de Santa Cantarina (UFSC), teve como tema "Geografia ou Geografias: Práxis Educativa e Formação Docente", e registrou 73 trabalhos publicados. Nesta edição os trabalhos foram divididos nos seguintes grupos: Formação Docente; Práticas de Ensino em Geografia na Escola e na Universidade; Pesquisa na Geografia Escolar; e Ensino de Geografia: Teorias e Metodologias.

Apesar de constarem publicados nos anais 73 trabalhos, para consulta só encontram-se disponíveis aqueles apresentados nos "Espaços de Diálogos e Práticas (EDPs)", considerados completos. Já os trabalhos apresentados em formato de painel, só é possível acesso ao título e não ao conteúdo. Desta forma, consideramos apenas os trabalhos completos, totalizando 49 trabalhos, dos quais 15 abordaram a temática físicaambiental, a saber: Mapas/Cartografia, Educação Ambiental, TIC/Geotecnologias, Vegetação e Geomorfologia, conforme Quadro 2 a seguir:

Quadro 2 - II ENPEG-SUL (2014).

\begin{tabular}{|c|c|}
\hline Tema abordado & № de trabalhos \\
\hline Mapas/Cartografia & 7 \\
\hline Educação Ambiental & 4 \\
\hline TIC/Geotecnologias & 2 \\
\hline Vegetação & 1 \\
\hline Geomorfologia & 1 \\
\hline
\end{tabular}

Fonte: Anais do II Encontro de Práticas de Ensino de Geografia da Região Sul, 2014.

Organização: Os autores.

Em 2017, foi realizada a terceira edição do ENPEG-SUL em Londrina/Paraná, na Universidade Estadual de Londrina (UEL). Nesta edição o evento foi realizado junto ao "XV Encontro de Ensino de Geografia, Mostra de Estágios e de Pesquisas: A formação

ParaOnde!?, Porto Alegre, v.12 n.2, p.107-114, 2019.http://seer.ufrgs.br/paraonde Edição Especial - III Colóquio de Pesquisadores em Geografia Física Ensino de Geografia 
geográfica na construção da cidadania". Assim como na segunda edição, os trabalhos foram divididos por grupos: Formação de professores e currículo da Geografia Escolar; Metodologia, didática e prática de ensino de Geografia; e Epistemologia da Geografia e Ensino.

Nesta edição foram publicados 59 trabalhos, dos quais 14 abordaram à temática física-ambiental, a saber: Mapas/Cartografia, Educação Ambiental, Paisagem, Clima, Sistema Solar, Geomorfologia, Bacias Hidrográficas, conforme Quadro 3 a seguir:

Quadro 3 - III ENPEG-SUL (2017).

\begin{tabular}{|c|c|}
\hline Tema abordado & No de trabalhos \\
\hline Mapas/Cartografia & 3 \\
\hline Educação Ambiental & 3 \\
\hline Paisagem & 3 \\
\hline Clima & 2 \\
\hline Sistema Solar & 1 \\
\hline Geomorfologia & 1 \\
\hline Bacias Hidrográficas & 1 \\
\hline
\end{tabular}

Fonte: Anais do XV Encontro de Ensino de Geografia, Mostra de

Estágios e de Pesquisas, 2017.

Organização: Os autores.

Recentemente, em junho de 2018, foi realizada a quarta edição do ENPEG-SUL em Florianópolis/Santa Catarina, na Universidade do Estado de Santa Catarina (UDESC), com o tema "Ensino de Geografia e Políticas Curriculares". Os trabalhos foram divididos nos seguintes grupos: Formação de professores, diretrizes e propostas curriculares; Práticas de ensino contemporâneas; e O conhecimento da Geografia escolar e suas diferentes linguagens.

Nesta edição foram publicados 73 trabalhos, dos quais 26 abordaram à temática física-ambiental, a saber: Mapas/Cartografia, TIC/Geotecnologia, Educação Ambiental, Paisagens Naturais, Geografia Física (conceitos gerais) e Geomorfologia, conforme Quadro 4 a seguir:

Quadro 4 - IV ENPEG-SUL (2018).

\begin{tabular}{|c|c|}
\hline \multicolumn{2}{|c|}{ IV ENPEG-SUL } \\
\hline Tema abordado & № de trabalhos \\
\hline Mapas/Cartografia & 10 \\
\hline TIC/Geotecnologia & 7 \\
\hline Educação Ambiental & 6 \\
\hline Paisagens Naturais & 1 \\
\hline $\begin{array}{c}\text { Geografia Física (Conceitos } \\
\text { gerais) }\end{array}$ & 1 \\
\hline Geomorfologia & 1 \\
\hline
\end{tabular}

Fonte: Anais do IV Encontro de Práticas de Ensino de Geografia da Região Sul, 2018.

Organização: Os autores.

ParaOnde!?, Porto Alegre, v.12 n.2, p.107-114, 2019.http://seer.ufrgs.br/paraonde Edição Especial - III Colóquio de Pesquisadores em Geografia Física Ensino de Geografia 
Entre a primeira e a quarta edição do Encontro de Práticas de Ensino de Geografia da Região Sul, foram publicados 245 trabalhos, dos quais 82 ligados à temática físicaambiental. Julgamos importante também analisar de qual instituição 0 trabalho/pesquisador é originário, para assim tentar detalhar melhor de onde vêm as pesquisas ligadas a esta temática dentro do ensino de Geografia na região sul do país. Dos 82 trabalhos, conseguimos identificar a origem de 79, conforme Quadro 5 a seguir:

Quadro 5 - Instituições as quais pertencem ostrabalhos/pesquisadores.
\begin{tabular}{|c|c|}
\hline Instituição de Origem & No de trabalhos \\
\hline UEL & 14 \\
\hline UDESC & 12 \\
\hline UFRGS & 9 \\
\hline UFSC & 9 \\
\hline UFPR & 8 \\
\hline UFSM & 7 \\
\hline UFFS & 6 \\
\hline UNIOESTE & 3 \\
\hline UFPel & 3 \\
\hline UPF & 1 \\
\hline ULBRA & 1 \\
\hline PUC/RS & 1 \\
\hline UNIRITTER & 1 \\
\hline USP & 1 \\
\hline UFV & 1 \\
\hline La Salle & 1 \\
\hline IFC & 1 \\
\hline Organização: Os autores. & \\
\hline
\end{tabular}

\section{Considerações finais}

Tivemos alguns problemas quanto à metodologia adotada, uma vez que as publicações das quatro edições do evento apresentavam diferentes critérios e padrões aceitos para publicação de trabalhos nos anais. Assim, fomos remodelando a metodologia de forma a contemplar nosso objetivo.

Ao analisarmos o número de publicações, verificamos que na primeira edição do ENPEG-SUL (2013) a porcentagem de trabalhos relativos à temática física-ambiental obteve uma representatividade mais expressiva, se comparada às outras edições, representando 42,2\% do total de trabalhos publicados. Nas edições seguintes, $2014 \mathrm{e}$ 2017, esta expressão diminuiu para $30,6 \%$ e $23,7 \%$ respectivamente, enquanto que na edição de 2018 este número voltou a crescer e representou $35,6 \%$ do total de trabalhos publicados.

Analisando as temáticas tratadas, verificamos que nas quatro edições as mais apresentadas/publicadas foram: Mapas/Cartografia, Educação Ambiental e TIC/Geotecnologias. Historicamente, a Educação Ambiental é um dos primeiros temas

ParaOnde!?, Porto Alegre, v.12 n.2, p.107-114, 2019.http://seer.ufrgs.br/paraonde Edição Especial - III Colóquio de Pesquisadores em Geografia Física Ensino de Geografia 
ligados ao Ensino de Geografia a surgir dentro da temática física-ambiental dos Programas de Pós-Graduação em Geografia, talvez por isso é um dos temas mais abordados. As preocupações com os mapas e a cartografia é um assunto mais recente, que vem ganhando destaque nas pesquisas nos últimos anos, assim como as Tecnologias de Informação e Comunicação bem como as Geotecnologias.

Quando analisamos as produções de acordo com a origem da pesquisa, é importante ressaltar que isto é em relação às publicações nos anais do ENPEG-SUL e não referente às pesquisas em ensino de Geografia no Sul do Brasil de uma forma geral. É importante destacar que além das 15 diferentes instituições do Sul do Brasil existem também as instituições vindas de outras regiões, tais como a USP e a UFV.

Algumas instituições, como por exemplo, a UEL, apesar de apresentarem um número significativo de pesquisas em relação às outras instituições, só participou com publicações no evento realizado em sua instituição, o que denota que das 14 publicações ligadas a UEL, 13 foram publicadas na edição do evento realizado em Londrina, contudo, também precisamos considerar a questão da logística que, muitas vezes, influenciam na participação mais efetiva dos pesquisadores nos eventos.

Desta forma, acreditamos que a pesquisa se justifica por evidenciar o debate que vem acontecendo nos eventos acerca do Ensino de Geografia, e neste caso, acerca das pesquisas ligadas à temática física-ambiental. Assim, esperamos que o trabalho aqui apresentado contribua com as discussões neste sentido.

\section{REFERÊNCIAS}

CALLAI, H.C. O ensino de Geografia nos trabalhos apresentados no XI ENANPEGE. Revista da Associação Nacional de Pós-Graduação e Pesquisa em Geografia (ANPEGE). v.12. n.18, especial GT Anpege, p. 43-55, 2016.

ENCONTRO DE ENSINO DE GEOGRAFIA, MOSTRA DE ESTÁGIOS E DE PESQUISAS, 15., 2017, Londrina. Anais eletrônicos... Londrina: UEL, 2017. Disponível em <http://www.uel.br/eventos/ensinogeo/arquivos/anais_ encontrogeo_2017_2.pdf>. Acesso em: 23 de mai. de 2018.

ENCONTRO DE PRÁTICAS DE ENSINO DA REGIÃO SUL, 1., 2013, Porto Alegre. Anais eletrônicos... Porto Alegre: AGB, 2013. Disponível em <http://enpegsul.wixsite.com/enpegsul/apresentaes-de-trabalho>. Acesso em: 23 de mai. de 2018.

ENCONTRO DE PRÁTICAS DE ENSINO DE GEOGRAFIA DA REGIÃO SUL, 2., 2014, Florianópolis. Anais eletrônicos... Florianópolis: NEPGeo, 2014. Disponível em <http://anaisenpegsul.paginas.ufsc.br/edicao-atual>. Acesso em: 23 de mai. de 2018.

4., 2018, Florianópolis. Anais eletrônicos... Florianópolis: UDESC, NEPED, 2018. Disponível em <https://enpegsuludesc.wordpress.coml>. Acesso em: 6 de ago. de 2018.

FREITAS, A.S.F. A formação inicial de professores de Geografia nos Encontros Nacionais de Pós-Graduação em Geografia. In: Encontro Nacional da ANPEGE, 21., 2015,

ParaOnde!?, Porto Alegre, v.12 n.2, p.107-114, 2019.http://seer.ufrgs.br/paraonde Edição Especial - III Colóquio de Pesquisadores em Geografia Física Ensino de Geografia 
Presidente Prudente. Anais Eletrônicos... Presidente Prudente: UNESP, 2015. Disponível em <www.enanpege.ggf.br/2015>. Acesso em: 20 de mai. de 2018.

FONSECA, R.L.; SALVI, R.F.; TORRES, E.C. O estado da arte das pesquisas em dissertações de mestrado em ensino de Geografia: contribuição para uma avaliação a partir das pesquisas nos programas nacionais stricto sensu (2008 - 2012). Revista da Associação Nacional de Pós-graduação e Pesquisa em Geografia (ANPEGE). v. 10. $n$. 13. p.141-163, jan-jun.2014. 